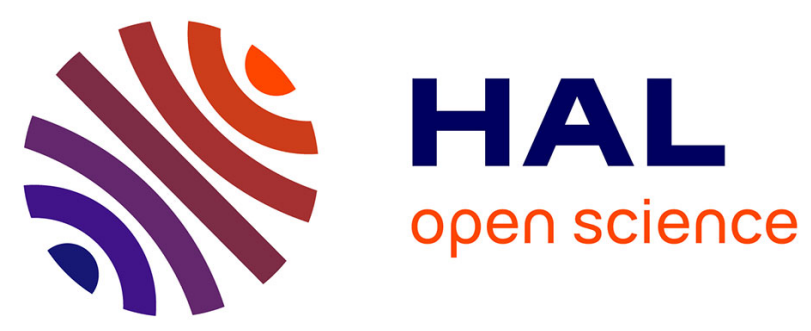

\title{
Pressure-Based Gain Factor Control for Mobile 3D Interaction using Locally-Coupled Devices
}

\author{
Lonni Besançon, Mehdi Ammi, Tobias Isenberg
}

\section{To cite this version:}

Lonni Besançon, Mehdi Ammi, Tobias Isenberg. Pressure-Based Gain Factor Control for Mobile 3D Interaction using Locally-Coupled Devices. CHI 2017 - ACM CHI Conference on Human Factors in Computing Systems, May 2017, Denver, United States. pp.1831-1842, 10.1145/3025453.3025890 . hal-01436172

\section{HAL Id: hal-01436172 \\ https://hal.inria.fr/hal-01436172}

Submitted on 18 Jan 2017

HAL is a multi-disciplinary open access archive for the deposit and dissemination of scientific research documents, whether they are published or not. The documents may come from teaching and research institutions in France or abroad, or from public or private research centers.
L'archive ouverte pluridisciplinaire HAL, est destinée au dépôt et à la diffusion de documents scientifiques de niveau recherche, publiés ou non, émanant des établissements d'enseignement et de recherche français ou étrangers, des laboratoires publics ou privés. 


\section{Pressure-Based Gain Factor Control for Mobile 3D Interaction using Locally-Coupled Devices}

\author{
Lonni Besançon \\ University Paris Saclay \\ Orsay, France \\ lonni.besancon@gmail.com
}

\author{
Mehdi Ammi \\ Limsi CNRS \\ Orsay, France \\ mehdi.ammi@limsi.fr
}

\author{
Tobias Isenberg \\ Inria \\ Orsay, France \\ tobias.isenberg@inria.fr
}

\begin{abstract}
We present the design and evaluation of pressure-based interactive control of 3D navigation precision. Specifically, we examine the control of gain factors in tangible 3D interactions using locally-coupled mobile devices. By focusing on pressure as a separate input channel we can adjust gain factors independently from other input modalities used in 3D navigation, in particular for the exploration of 3D visualizations. We present two experiments. First, we determined that people strongly preferred higher pressures to be mapped to higher gain factors. Using this mapping, we compared pressure with rate control, velocity control, and slider-based control in a second study. Our results show that pressure-based gain control allows people to be more precise in the same amount of time compared to established input modalities. Pressure-based control was also clearly preferred by our participants. In summary, we demonstrate that pressure facilitates effective and efficient precision control for mobile 3D navigation.
\end{abstract}

\section{ACM Classification Keywords}

H.5.2. User Interfaces: Input devices and strategies

\section{Author Keywords}

Pressure input; tangible interaction; TUI; 3D navigation.

\section{INTRODUCTION}

We present the design and the evaluation of a prototype that adds pressure-based input sensing to the back of a mobile device. Pressure or isometric force is a continuous form of input that is increasingly used in HCI systems. The recent release of devices equipped with $3 D$-touch ${ }^{1}$ may well encourage an even higher number of manufacturers to provide to equip their systems with this input channel-so our research enables future developers to design pressure-based control effectively.

We use pressure as an input channel to provide users with a manual control of the gain factor associated with the tangible

\footnotetext{
${ }^{1}$ https://developer.apple.com/ios/3d-touch/
}

Permission to make digital or hard copies of all or part of this work for personal or classroom use is granted without fee provided that copies are not made or distributed for profit or commercial advantage and that copies bear this notice and the full citation on the first page. Copyrights for components of this work owned by others than ACM must be honored. Abstracting with credit is permitted. To copy otherwise, or republish, to post on servers or to redistribute to lists, requires prior specific permission and/or a fee. Request permissions from permissions@acm.org.

CHI 2017, May 6-11, 2017, Denver, CO, USA.

Copyright is held by the owner/author(s). Publication rights licensed to ACM. ACM ISBN 978-1-4503-4655-9/17/05 _..\$15.00.

http://dx.doi.org/10.1145/3025453.3025890 manipulations of 3D content shown on the device. Gain factors (also called control-display gain, CD gain; $[8,36,56])$ play an important role in interaction. Hinckley et al. [25] even state that one of the major hurdles with 3D interaction is to be able to "provide an interface which effectively integrates rapid, imprecise [...] object placements with slower but more precise object placement, while providing feedback that makes it all comprehensible." Past work has focused on techniques to set the CD gain for mouse or tactile interaction (e. g., [6, 13, 36, $56])$, but some work for direct 3D input [30,31] and interaction in VR [22] exists as well. In the special case of tangible manipulations, different gain factor for the same interaction mapping can make a manipulation extremely exhausting and frustrating (e.g., when the gain factor is low and the user has to make many different arm/hand/shoulder movements) or really frustrating (e.g., when the gain factor is high and the manipulation not precise enough).

Previous work in the context of 3D manipulations [7] has shown that tactile manipulation of a slider widget during tangible manipulation was not appropriate: even though users could precisely set the gain factor, they did not want to stop their current interaction to reset the gain factor. With our pressurebased control we present a new approach to control the gain factor independently from the other input modalities.

Our contributions are thus threefold. First, we present the design of a back-of-device pressure-sensing system controlling the gain factor associated with tangible manipulation of a mobile device. Second, we study the pressure-based control of gain factors to learn which interaction mapping is preferred by participants and used most efficiently by them. Finally, we compare this pressure-based form of gain control with established mappings including velocity-based, slider-based, and rate control. Our evaluation comprises performance workload and fatigue measures as well as subjective preferences. Our results show that pressure-based control was not only clearly preferred but also that pressure-based gain control allows people to be more precise in the same amount of time compared to established input modalities.

\section{RELATED WORK}

Work related to our own comes from one of three major fields: interaction with the back of (mobile) devices, the use of pressure as an input modality, and the use of pressure to augment other input techniques. We discuss these aspects next. 


\section{Back-of-Device Interaction}

Several research projects have investigated interaction on the back of the device as a way to eliminate on-screen occlusion. For instance, systems like BehindTouch [26] and BlindSight [34] use a 12-key pad on the back of a mobile device. In particular for small devices, occlusion is one of the biggest usability issues. In the context of very small screens, Baudisch and Chu [5] as well as Wigdor et al. [52] thus propose to combine back-of-device interaction with a see-through effect to improve pointing. Liang et al. [35] also propose to use a secondary mobile device attached to the the back of the first one to facilitate tactile input above and under the mobile device and thus support task such as rotation, translation, stretching or slicing. Similarly, Shen et al. [47] propose a set of gestures for 3D interaction with back-to-back devices. In addition, back-of-device interaction has been used on larger devices. For instance, Wigdor et al. [53] propose to use a two-sided interactive touch table to add a new dimension of input for co-located collaborative work. Finally, back-of-device interaction has also been used combined with other types of input; e. g., with stylus input on a PDA [49] or to detect bending using a position sensor on the back of the Gummi device [45]. We build on the general approach of back-of-device input to provide easily accessible pressure control when interacting while holding a tablet.

\section{Pressure as an Input Modality}

Pressure input is a continuous input data $[12,14,43,48]$ that has been shown in the literature to be usable as a primary modality for a variety of tasks. Early work conducted by Buxton et al. [12] includes an example of a drawing application. Buxton et al. map continuous pressure data to a continuous scale that varies the width of the painting brush, allowing users to control both width and path with a single finger. Brewster et al. [9], for instance, use pressure to control text entry. They map the pressure input to two discrete states: a light pressure will be mapped to lowercase letters while a strong pressure will be mapped to uppercase letters. Similarly, Mclachlan et al. [38] and Wilson et al. [54] use pressure-based menu selection on mobile devices. Ren et al. [44] use pressure values of penbased interfaces to control the continuous size of a circular cursor (or its contexts) to assist users for selection tasks. Other non-touch-enabled devices also make use of pressure as a primary input. For instance, Issartel et al. [29] use pressure input to realize a grasping metaphor on a tangible volume. In their case, pressure controls a state representing whether the user is trying to grasp - for pressure larger than a given threshold - or release a virtual object. In our work we map the pressure input to a continuous range of gain-factor values.

Pressure input can be captured by means of force-sensing resistors (FSRs) [38] or can be indirectly captured on touch screens. One of the techniques used for this purpose builds on the fact that a higher pressure on a point leads to a wider point of contact between the finger and the surface [11] and was implemented by Forlines and Shen [21] or Benko et al. [6]. Arif and Stuerzlinger [2], for instance, use it to create a technique to bypass incorrect word predictions of text entries on a tactile device. While this indirect sensing of pressure on touch-screen is promising, in our work we only consider sensor-captured pressure. Indeed, even though our prototype has a touch screen, we take advantage of back-of-device pressure sensing which is easier to achieve with simple FSRs.

A vast majority of studies conducted on pressure in the HCI community have focused on determining how many distinct levels of pressure can be applied by users. Early work conducted by Herot and Weinzapfel [24] already suggested that accuracy with pressure input methods is achievable with a continuous and real-time visual feedback. With the presence of a visual feedback, pressure-based interaction has been proven to be highly accurate. For instance, Cechanowicz et al. [14] proved that users could differentiate between 64 modes on a dual-pressure augmented mouse, while Wilson et al. [54] showed that users could distinguish accurately up to 10 levels of pressure with adequate feedback on a mobile device. The value obtained in the former is high enough for us to consider it as a virtual continuous scale. The value obtained in the latter can still be seen as close to continuous for our purposes. Indeed, participants in our study had to use pressure input to vary the gain factor from 0.3 to 3 (i. e., by a factor of 10).

\section{Pressure as Augmentation}

Pressure can be a direct and primary way to interact on mobile devices and thus can replace touch interaction. We are investigating, however, the use of isometric force as a supplementary, auxiliary input that could augment or complete other input technique. Touch and pressure input have been combined in the past, for example, by Arif et al. [1] to increase the security of conventional digit-lock of recent smartphones. McLachlan et al. [37] investigated this characteristic of the pressure input in the context of bimanual interactions on mobile devices. They could not find evidence that would suggest that the pressure input had effects on the accuracy of the dominant-hand performing touch inputs. Similarly, McLachlan and Brewster [38], demonstrated that the ability to perform simultaneous pressure inputs and touch gestures depended on the complexity of the gesture. Tangible 3D manipulations are regarded as natural since they are based on skills people have developed through their everyday interactions [27]. We thus hypothesize that these results may be generalizable to tangible 3D manipulations of a mobile devices and that thus the pressure input facilitates an additional and independent form of control to be used to adjust the gain factor in 3D interactions. Ramos and Balakrishnan [42] proposed such a combination of pressure and touch input. The first modality is used to provide a fluid integrated manipulation of the scale while the touch input is used to provide parameter manipulation within the pressureobtained scale. Similarly, Ramos et al. [43] combined position (obtained via touch input) and continuous pressure input (obtained from a stylus) to provide Pressure Widgets on mobile devices. Our design builds on their ideas but we use pressure to adjust the gain factor, while 3D navigations are still carried through physical manipulation of the tablet.

\section{PROTOTYPE}

To test this interaction concept we used an existing mobile device as a locally-coupled ${ }^{2}$ tangible $3 \mathrm{D}$ exploration tool and

\footnotetext{
${ }^{2} \mathrm{~A}$ locally-coupled device is both display and input device [30, 41].
} 

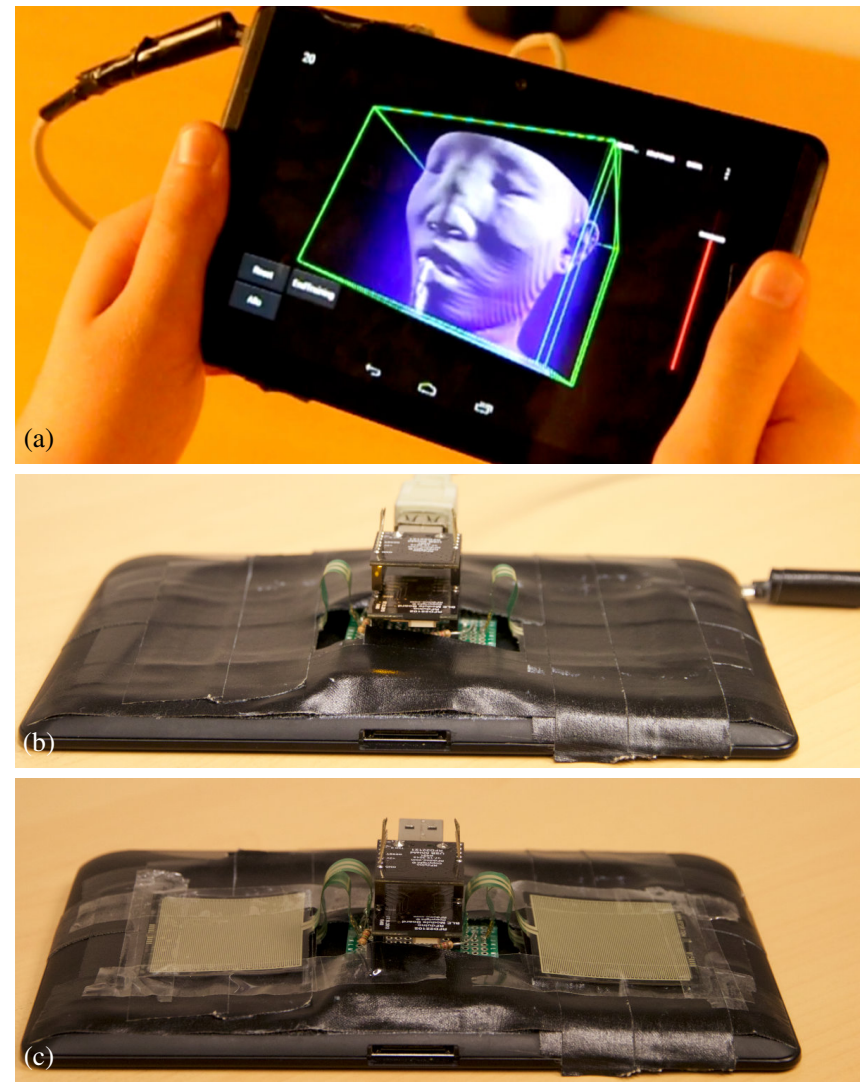

Figure 1. Interaction prototype: (a) prototype in use, (b) electronics installation on the back with taped pressure sensors, and (c) mock-up of the arrangement of the pressure sensors (hidden by the tape in (b)).

fitted it with pressure sensors (see Fig. 1). We used a Google Tango tablet ${ }^{3}$ as it provides both a tactile screen and a positionaware mechanism that facilitates tangible manipulations. We then augmented the tablet with back-of-device pressure FSR sensors that are located right under users' fingers on the back of the tablet. The FSR sensors are often used in pressuresensing prototype [37] and we coupled them to a RFDuino board, each one of them with a $3.3 \mathrm{k} \Omega$ resistance. The sensitivity of the FSRs depends on the value of the resistance and a pilot study with six participants allowed us to determine that $3.3 \mathrm{k} \Omega$ was ideal in our case. To keep the prototype fully portable, the RFDuino board is powered by a cable that connects it to the micro-USB port of the tablet. The pressure values are computed by the RFDuino board and sent over Bluetooth Low Energy (BLE) to the tablet. Clutching is achieved by putting/removing the fingers from the pressure sensors Fig. 2. While we first wanted to realize the clutching through touches on the screen, another pilot study showed us that it was easier for people to clutch with the pressure sensors. Because previous work $[14,48,54,55]$ has shown that pressure input is made more precise with the help of visual feedback, a cursor (aka slider) was added to the GUI on the tablet to reflect the gain-factor value obtained with pressure input. This kind of visual feedback has been used before in

\footnotetext{
${ }^{3}$ https://get.google.com/tango/
}

various studies and setups such as the ones used by McLachlan et al. [37, 38].

\section{EXPERIMENT 1: CHOICE OF FORCE MAPPING}

We conducted a first study to compare two possible pressurebased gain factor adjustment techniques. The first technique (P1) maps the pressure of the sensors directly to the gain factor: a high pressure results in a higher gain factor, i. e., to larger/stronger motions. The metaphor for this mapping is that the stronger pressure forces are equivalent to stronger and larger motions, and that lower pressure thus yields a more precise control. The second technique (P2) does exactly the opposite: a high pressure results in a lower gain factor, i. e., to more precise motions. The metaphor of this second technique is that with stronger pressure the interacting person holds on more tightly to be able to better control a precise manipulation. Our goal with this experiment was to determine whether one of these two technique yield better results and/or is preferred by users and thus to to determine which mapping we should use in our follow-up experiment.

\section{Participants}

For this first study we recruited 12 unpaid participants ( 2 female; ages 21-39, mean $=26.75$, med $=26, \mathrm{SD}=5.01)$. Six of them had at least university degree, while the remaining six had at most an A-level equivalent. Five of of the participants were used to 3D manipulation through the extensive use of video games or 3D software. All participants were right handed and had normal or corrected-to-normal vision.

\section{Procedure and Task}

We first presented participants with the tablet device and told them they would have to perform translation and rotations in a 3D virtual world. We presented them the application and showed how the clutching was achieved. An initial docking target was already present during this training phase of the experiment. We asked participant to try and use the tablet so as to match the target docking. The purpose of the training was twofold. First, as highlighted by Issartel et al. [28], we wanted to assert whether the participant preferred allocentric or egocentric mapping. ${ }^{4}$ Because our docking task was similar to the first environment used by Issartel et al. [28] which showed a $70 \%$ preference for the egocentric mapping, we set the initial mapping to egocentric. However, this initial training could be used to set it to allocentric if participant felt that they could not use the mapping correctly. Four participants did so and set their mapping to allocentric. Second, the gain factor was set by the experimenter for the initial training once to both a high value (i.e., 3) and to a low value (i. e., 0.3) - to let our participant understand the need for a manual control of the gain factor. Achieving a precise positioning was almost impossible with the high gain factor because it was too sensitive, while it took more than a minute for participant to do

\footnotetext{
${ }^{4}$ These two notions are frequently discussed in the literature [10, 32, 40]. In general, the egocentric term seems to be associated with the idea of the viewing perspective, and the allocentric term with the idea of a fixed, external reference point. In other words, when the manipulated object is being moved in the same direction as the tablet, the mapping is allocentric. When the manipulated object moves in the opposite direction of the tablet, the mappings is egocentric.
} 


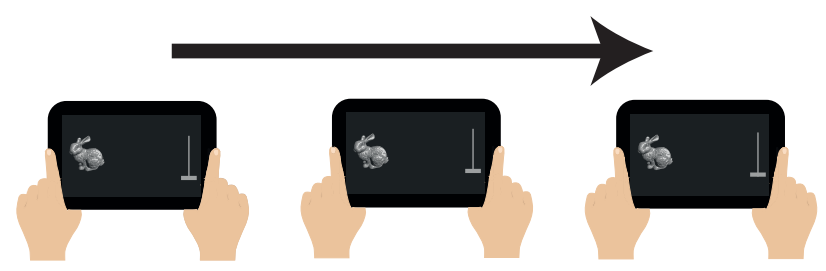

(a)

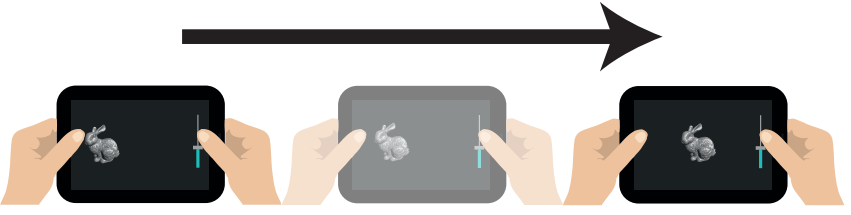

(c)

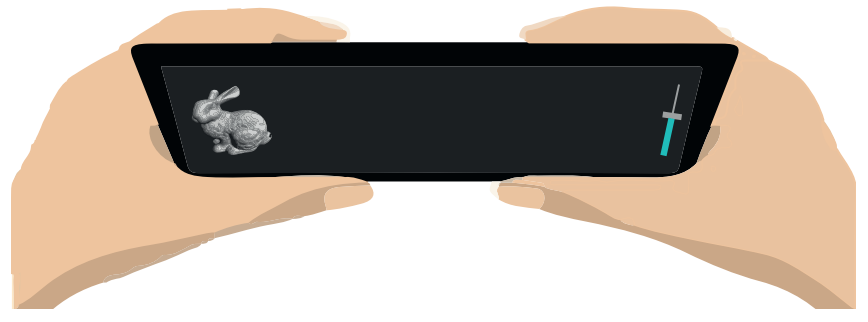

(b)

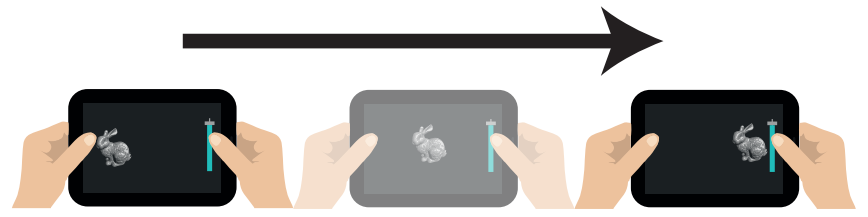

(d)

Figure 2. Illustration of the clutching mechanism: (a) fingers are not on the pressure sensors, thus movements sensed by the tablet are not propagated to the virtual object; (b) the force applied to the pressure sensors is mapped to the slider such that movements are now propagated to the virtual object; (c) if the pressure is low the slider value and the gain factor value are low such that the virtual object is translated only by a little; (d) if the pressure is high the slider value and the gain factor value are high such that the virtual object is translated more than in (c).

it with a low gain factor. Each participant thus experienced both extreme gain factors, in a counter-balanced order. During training, participants had an unlimited time to get used to the manipulation of the tablet and the purpose of the docking task.

Once they expressed to be familiar with the interaction techniques and task type, the experimenter launched the actual experiment. Participant were asked to complete 20 docking tasks for each technique (i. e., a total of 40 docking tasks), for each of which they had a maximum of 30 seconds. The order of technique was counter-balanced to avoid learning biases. The pool of possible docking targets had been manually created ahead of time to ensure that participants had to frequently manually change the gain factor. For each trial, the target was randomly picked from this pool and then removed from the pool. At the end of the experiment, each participant's preference was asked by the experimenter and a semi-structured interview was conducted to determine whether participant could use the technique properly.

\section{Hypotheses}

We hypothesized, based on previous work [37, 38], that (H1) the use of pressure as an input modality to control the interaction gain factor would not endanger the use of tangible control of 3D manipulations using the mobile device. We also hypothesized that (H2) it would be more natural for users to use P2 than it is to use $\mathrm{P} 1$ and that $\mathrm{P} 2$ would thus outperform $\mathrm{P} 1$-due to our own observations that people playing video games often put a lot of effort in being precise and, as they perform these precise interactions, tend to squeeze their game controller.

\section{RESULTS OF EXPERIMENT 1}

We collected a total of 480 docking trials from our 12 participants, i. e., 240 per technique. In addition, we recorded the answers of the participants in the semi-structured interview and analyzed our participants' subjective preferences and a comparison of their subjectively rated intuitiveness. First, to analyze our hypothesis $\mathrm{H} 1$, we specifically asked our participants during the semi-structured interviews about whether the additional pressure-input was harmful to their 3D manipulations. None of them reported so. We thus fond no evidence that would refute H1, making the pressure-based gain factor manipulation a viable option for tangible 3D interaction.

To assess the docking precision and thus to analyze $\mathrm{H} 2$, we then compared the Euclidean distance of and the angular difference between the manipulated object and the docking target. ${ }^{5}$ We first discuss the Euclidean and the angular distance to the target, for both technique. The Euclidean distance is computed as the distance between the centers of the two objects. The angular difference $d_{a}$ is computed as

$$
d_{a}=2 \cdot \arccos \left(q_{d \omega}\right) ; \quad q_{d}=q_{o}^{-1} \cdot q_{t}
$$

with $q_{o}$ being the quaternion of the manipulated object, $q_{t}$ being the quaternion of the target, thus $q_{d}$ being the difference quaternion, and $q_{\omega}$ being the $\omega$ component of an $\omega+x i+$ $y j+z k$ quaternion with $i^{2}=j^{2}=k^{2}=i j k=-1$. We then aggregated and averaged both the angular and the Euclidean distances per participant. The distribution is not normal, so we estimated population means using $95 \%$ bootstraped confidence intervals (CIs). Fig. 3 clearly shows that there is no evidence of a better performance of P2 over P1 for both the Euclidean distance and the angular distance to the target.

Even though we found no evidence of a performance difference between the two techniques, there were differences in

\footnotetext{
${ }^{5}$ Data from HCI experiments has often been analyzed by applying null hypothesis significance testing (NHST) in the past. This form of analysis of experimental data, however, is increasingly being criticized by statisticians $[4,16]$ and within $\mathrm{HCI}[18,19]$. Therefore, we report our analysis using estimation techniques with effect sizes and confidence intervals (i.e., not using $p$-values) as recommended by the APA [51]. The term effect size here simply refers to the measured difference of means-we do not make use of standardized effect sizes [15] because their use is not generally recommended [3]. While we make use of estimation techniques, a $p$-value-approach reading of our results can be done by comparing our CIs spacing with common $p$-value spacing as shown by Krzywinski and Altman [33].
} 

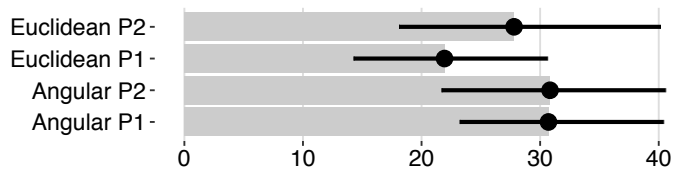

50

Figure 3. Euclidean and angular distance to the target for both P1 and P2. Error bars are $95 \%$ bootstrapped confidence intervals.

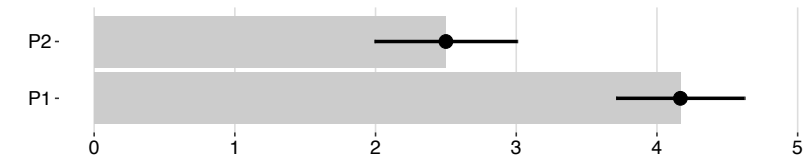

Figure 4. Likert scale score of intuitiveness for each technique. Error bars: $95 \%$ bootstrapped CIs.

preference. Fig. 4 shows aggregated ratings of the intuitiveness of each technique on a five-point Likert scale, and 11 out of our 12 participants reported that their favorite technique was P1 which maps a strong pressure input to a high gain factor. They all explained during the semi-structured interview that it felt more logical to have such a mapping. The remaining participant had no overall preference but stated that $\mathrm{P} 1$ also seemed more logical. Furthermore, four participants reported that P1 was less tiring than P2 and another six stated that P1 was easier to use. Finally, three participants reported a higher exit error issue-often found in tactile interaction [50]—with P2. Indeed, when leaving a state of high pressure to release the fingers and clutch, the gain factors gets from a very low value to a high value and any involuntary movement of the tablet at that time is thus followed by a relatively big movement of the manipulated object in the virtual world. For all the above reasons, we thus refuted hypothesis $\mathrm{H} 2$ and decided to use P1 as the primary mapping for the following experiment.

\section{EXPERIMENT 2: USABILITY OF GAIN FACTOR CONTROL}

Our second study used the same prototype and task as Experiment 1 . However, we asked participants to perform the docking task with one of four different techniques to compare our pressure-based technique to three established mappings: slider-based control, velocity-based control, and rate control. The first one used a touch-based slider on the dominant hand's side of the screen that allows users to manipulate the gain factor. Arguably, sliders are the most commonly used ways to provide values within a specific range in regular interfaces and, in contrast to keyboard input, are effective on mobile devices. We told each participant that they could change the placement of the slider to the left or right side regardless of their dominant hand. The second technique used the velocity of the tablet's movements (rotations and translations) to derive the gain factor-similar to mouse-based gain factor control $[13,20,22]$. The third technique used a rate-control approach: the further the tablet was translated/rotated away from its initial position, the higher was the gain factor for translation/rotations. However, because rate-control has been proven to be inappropriate for devices without a self-centering mechanism [57], we compensated by adding a centering mechanism based on clutching. Still, rate-control is frequently used for $3 \mathrm{D}$ games with remote controls or joysticks so we deemed it an appropriate candidate for a comparison with the added centering mechanism. For the sake of fairness, the value of the gain factor was represented on the (potentially inactive slider in all cases. We also note that the gain factor ranges were identical for all conditions. We used the pilot studies to find appropriate (linear) mappings from the available input value ranges of these three techniques to the range of gain factors.

\section{Participants}

For this study, we recruited 24 new unpaid participants ( 9 female; ages $20-53$, mean $=31.6$, med $=26.5, \mathrm{SD}=11.1$ ). Twelve of them had at least a university degree (bachelor or equivalent), while the remaining half had at most an Alevel equivalent. Half of them were experienced with 3D manipulation through extensive use of video games $(9 x)$ or $3 D$ modeling software $(3 \times)$. Two participants were left-handed and all participants had normal or corrected-to-normal vision.

\section{Procedure and Task}

This experiment was largely based on the precudure of Experiment 1, using the same docking task. Participants were first greeted and introduced to the tablet device, before being told that they would perform translations and rotations with it. We explained the clutching mechanism and made them perform the docking with the high and low gain factors to allow them to understand the necessity of a manual gain factor control. We asked participants to complete 20 docking tasks with each of the mentioned 4 different techniques (total of 80 docking tasks). For each docking task we allowed participants up to 20 seconds, a time span that is based on the average time of 19.2 seconds it took participants to complete the task in Experiment 1. The second experiment thus lasted a bit more than 26 minutes overall.

Similar to Experiment 1, the second study also used an egocentric mapping but participants could change it during this training phase. Eleven participants stated that this mapping was not completely natural for them and switched to an allocentric mapping. In between each technique, participants were asked to fill in a questionnaire to assess their workload and their fatigue. For the former, we used NASA's Task Load Index (TLX). ${ }^{6}$ For the latter, we created our own questionnaire based on Shaw's approach [46]. To avoid seemingly random choices made in the second part of the TLX (which were often seen as confusing by participants in our pilot studies) that would lead to inconclusive or even incorrect results, we removed the second part of the TLX questionnaire. We thus performed a $R A W T L X$ (RTLX) which, according to Hart's survey [23], is equally well suited as a regular TLX. Finally, at the end of the experiment-and following Nielsen's [39] recommendation for the evaluation of subjective preferenceswe asked participants to rank each technique based on their preferences and we conducted semi-structured interviews.

To avoid participant response bias [17], we told our particpants that all the techniques were state-of-the-art techniques, that none of them was invented by us, and that we simply wanted to evaluate how they performed with each of them. Our participant number of 24 ensured that each sequence of conditions was tested exactly once in a counter-balanced fashion).

${ }^{6}$ http://humansystems.arc.nasa.gov/groups/tlx/downloads/TLXScale.pdf 
(a)

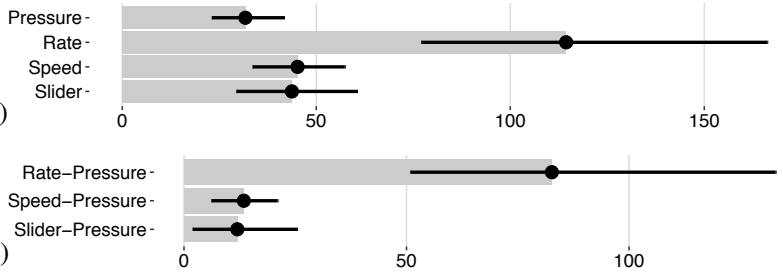

Figure 5. Euclidean distance to the target: (a) absolute values and (b) pair-wise differences. Error bars: $95 \%$ bootstrapped CIs.

\section{Hypotheses}

Based on pilot studies and previous work results, we formulated a number of hypotheses:

H3 Even with the clutching mechanism to reset the interaction center, participants' performances using rate control will be poor and it will not be preferred by our participants. The reason is that, even with an added clutching for recentering [57], on locally-coupled devices the interaction will not be natural due to the conflict of the rate control for the gain factor with the tangible position-based control of the 3D manipulation and it will thus not be understood correctly by most participants.

H4 Pressure-based control will have a higher performance (accuracy) than the three other techniques because it facilitates gain factor manipulation using a separate input channel that does not disrupt the tangible manipulation.

H5 Pressure-based control, however, will cause a higher fatigue in participants' fingers due to the additional force that is necessary compared to the other interaction techniques.

H6 Speed-based control will be the cause of a high overall fatigue and physical demand, even though the mental demand would be low compared to other techniques-we noticed that participants in our pilot studies tended to resort to overly fast movements to ensure that the gain factor will be high, causing sore arms and shoulders.

\section{RESULTS OF EXPERIMENT 2}

We now discuss the measured performance values in form of the Euclidean and the angular distance to the target. We also present participants' subjective preferences as well as their self-assessed fatigue, and workload. Similar to Experiment 1 we report our results using simple effect sizes.

\section{Euclidean and Angular Distances}

We collected a total of 1920 trials from our 24 partcipants, 480 per technique. We averaged our distance observations per participant and computed population means using $95 \%$ bootstrapped confidence intervals.

The Euclidean distances to the target for all four techniques is shown in Fig. 5(a). These results show strong evidence for a better performance of pressure-based, speed-based, and slider-based control compared to rate control. There is also weak evidence for a better performance of pressure-control over the slider-based and speed-based methods. To assess this difference in detail, we looked at the difference between pressure-control and the three other condition as shown in

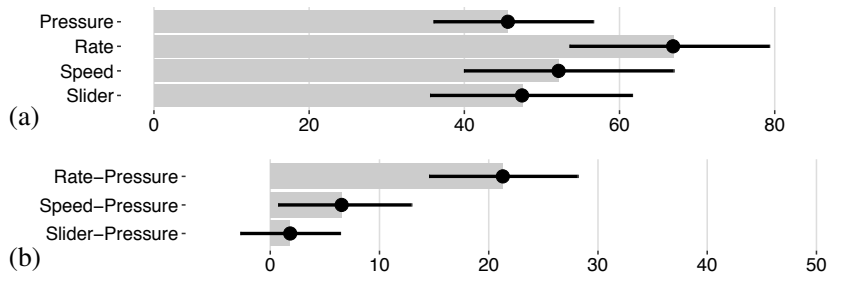

Figure 6. Angular distance to the target: (a) absolute values and (b) pairwise differences. Error bars: $95 \%$ bootstrapped CIs.

Fig. 5(b). The fact that none of the confidence intervals for these differences overlaps with zero supports our finding of pressure-based control of the gain factor being more accurate than the other techniques: it leads to 3.6 smaller Euclidean distances than rate-control, 1.4 smaller than speed-based control, and 1.3 smaller than slider-based control.

The angular distances to the target for all four techniques are shown in Fig. 6(a). Again, there is a strong evidence that rate control is outperformed by all three other techniques. While there is no evidence for a difference in performance between speed-based and slider-based control or pressurebased and slider-based control, there is slight evidence that pressure-based control may also perform better than speedbased control for rotational distances. Similar to the analysis of the Euclidean distance, we computed the pair-wise differences between pressure-based control and the other techniques and show them in Fig. 6(b). These values confirm that pressurebased control of the gain factor allowed participants to obtain a better angular accuracy than rate-control and speed-based controls The confidence interval of the difference between slider-based and pressured-based control, however, overlaps 0 so that we claim a difference between these two modalities.

\section{Workload}

The individual results of the TLX questionnaire are shown in Fig. 7. Fig. 7(a) suggest that strong evidence exists of speed-based control being physically more demanding than slider-based control, but we cannot make any further conclusion with respect to the other techniques. Fig. 7(b), however, shows strong evidence of rate control being approximately 1.5 times more mentally demanding that the other three techniques. Fig. 7(c) exhibits strong evidence of rate control being temporally more demanding than pressure-based, speed-based, and slider-based control. We conjecture that this observation results from participants being more stressed with a technique that they did not master, thus suggesting that rate control does not give them the level of control they wanted. This hypothesis is further reinforced by the confidence intervals shown in Fig. 7(d) which provide strong evidence for rate control giving a much higher (at least twice and almost three times as much when compared to pressure-based control) perceived performance than the other three conditions. Fig. 7(d) also provides strong evidence of a better perceived performance for pressure-based control than for the slider-based or speed-based conditions. While there is no evidence for an difference in effort between rate control, speed-based, or slider-based control, Fig. 7(e) provides strong evidences of pressure-based control being less effort-demanding than the three other techniques. 
(a)

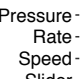

(c)

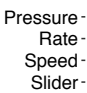

(e)

Rate-
ressure-

Speed-
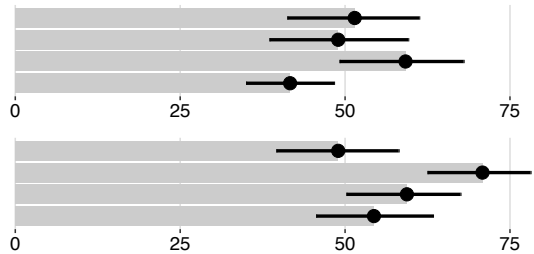

75
100

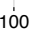

$10 \dot{0}$ (b)

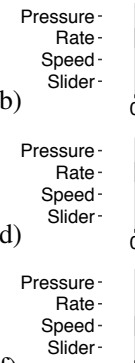

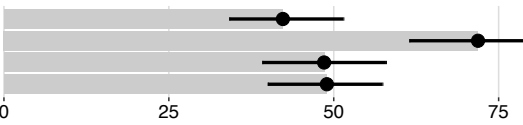

75

(f)

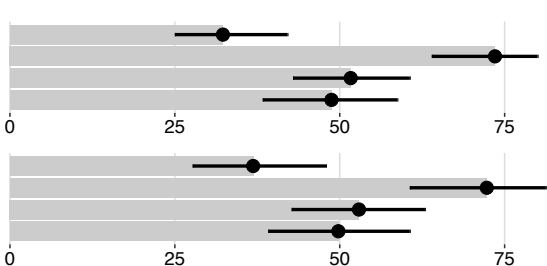

100

Figure 7. Workload measurement in NASA TLX units $(\in[0,100])$ with respect to (a) physical demand, (b) mental demand, (c) temporal demand, (d) performance (lower is better), (e) effort, and (f) frustration. Error bars: $95 \%$ bootstrapped CIs.

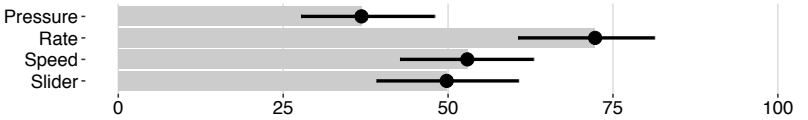

Figure 8. Total workload per factor. Error bars: $95 \%$ bootstrapped CIs.

Similarly, Fig. 7(f) has strong evidence that pressure-based control is less frustrating by a factor of two than rate control, and strong evidence for speed-based and slider-based control to be less frustrating than rate control.

The overall TLX workload is shown in Fig. 8. There is strong evidence for a higher workload of the use of rate-control compared to the other techniques. While we cannot find evidence for differences in workload between speed-based and sliderbased control, there is evidence that pressure-based control is overall less demanding than all the other techniques.

\section{Fatigue}

Fig. 9 presents the results of the fatigue questionnaire that each participant filled in after each condition. We used a 11-point Likert scale (0 meaning no fatigue at all and 10 meaning extreme fatigue) for fingers, hands, arms, and shoulders. Fig. 9(a) suggest that there is strong evidence of the pressure-based control being slightly more tiring (between 1.3 and 1.5 times) for the fingers than the other three techniques. It appears, however, that the overall finger fatigue caused by a pressurebased control is still not too high. We also can see in Fig. 9(b) that all techniques result in a similar hand fatigue. Fig. 9(c) and Fig. 9(d) provide strong evidence of speed-based control causing more arm and shoulder fatigue than pressure-based and slider-based control. However, there is no evidence of a fatigue difference between rate control and the other three conditions for arms and shoulder. The total fatigue measurements are shown in Fig. 10. Fig. 10(a) treats all four fatigue aspects equally and derives the total fatigue measurements as a sum of the individual factors. From these sums we cannot find evidence for a difference in the overall fatigue measure between the different technique. However, the different fatigue aspects may be more or less important to people, so we also asked our participants about the importance of the individual fatigue aspects and derived a weighted aggregated fatigue rating in Fig. 10(b). The mean weighted aggregated fatigue rating is lowest for pressure-based control, with weak evidence of it being different from speed-based control.

\begin{tabular}{l|ccc|rrrr}
\hline technique & median & mean & $S D$ & $\# 1^{\text {st }}$ & $\# 2^{\text {nd }}$ & $\# 3^{\text {rd }}$ & $\# 4^{\text {th }}$ \\
\hline pressure & 1.0 & 1.5 & 0.8 & 15 & 7 & 1 & 1 \\
rate & 4.0 & 3.4 & 1.0 & 3 & 0 & 3 & 18 \\
speed & 3.0 & 2.6 & 0.8 & 3 & 8 & 12 & 1 \\
slider & 2.5 & 2.4 & 0.9 & 3 & 9 & 8 & 4 \\
\hline
\end{tabular}

Table 1. Participants' preferences between their most favorite (1) and least favorite (4) technique to control the gain factor.

\section{Preference and Qualitative Feedback}

After the experiment, we asked participants to rank the technique from their preferred (1) to their least preferred (4) method for which we present the results in Table 1. The pressure modality was most preferred $15 \times$ and the slider-based control, the speed-based control, and the rate-control were most preferred by 3 participants each. These results show a strong preference for the pressure-based control of the gainfactor. Similarly, we found that most participants did not like the rate control technique. It is more difficult, however, to state a definite difference between speed-based and and sliderbased control. To better analyze this result, we determined the number of times each technique was picked as the favorite with simultaneous confidence intervals (that are applied on a multinomial distribution) and show the result in Fig. 11. The non-overlapping confidence interval of pressure-based control with all three other techniques allows us to infer that pressurebased control is likely to be the preferred technique by a vast majority of the population.

Participants also voiced interesting comments during the study. Two participants stated that the pressure-based control gave them a better feeling of precision and of being in control, thus "eliminating all the temporal pressure of the experiment." Two other participants also reported that, although the "speed-based control [was] interesting," it was difficult to evaluate and find the correct speed needed to achieve what they wanted. Three participants who picked the slider as their favorite technique stated at the end of the experiment that it was "easy to use" and that it gave "the more precise control of the gain factor."

\section{DISCUSSION}

Based on these results we now discuss our most relevant and interesting findings. Our ultimate goal in this work is to find a modality that is suitable for gain factor control for 3D tangible manipulations of a locally-coupled device. 
(a)

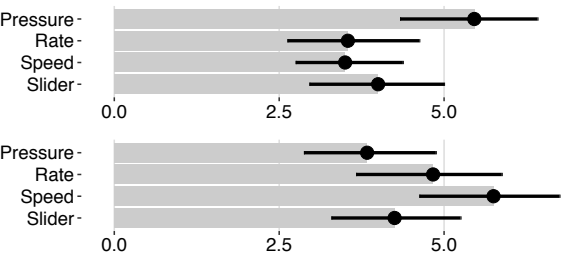

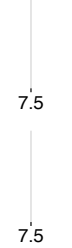

(b)

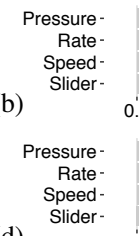

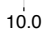

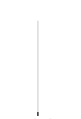

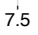

(d)

7.5

10.0

10.0

0.0

2.5
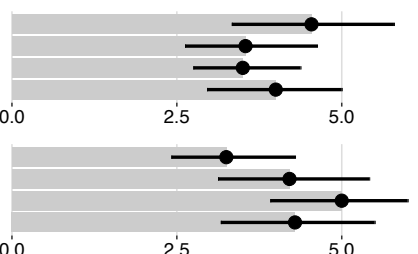

Figure 9. Fatigue measurement on a scale from 0 to 10 for (a) fingers, (b) hands, (c) arms, and (d) shoulders. Error bars: $95 \%$ bootstrapped CIs.

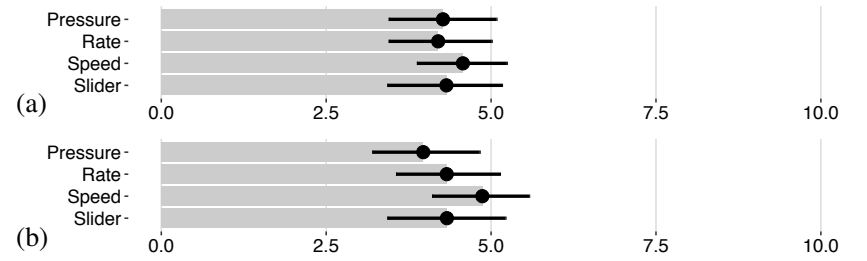

Figure 10. Aggregated fatigue measurements: (a) non-weighted and (b) weighted, both from 0 to 10 . Error bars: $95 \%$ bootstrapped CIs.

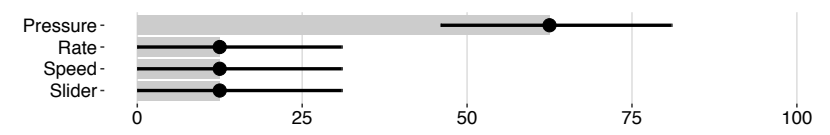

Figure 11. Percentage of times of each technique to be named the number one favorite. Error bars: $95 \%$ bootstrapped CIs.

\section{Performance}

Support for our hypothesis H3 comes from the poor performance of rate control compared to the other three techniques. For both Euclidean and angular distances, rate control exhibits performance results that do not make it a viable technique for gain factor manipulations. One of the reasons for this finding is likely the lack of self-centering mechanism [57]. However, an interaction technique based on rate control is also an indirect and joystick-like manipulation which contrasts the direct manipulations otherwise offered by the tablet. This contrast may also contribute to the poor performance of rate control we observed with our participants.

For both Euclidean and angular distances, our study did not reveal a difference between speed-based and slider-based control. Both techniques also performed noticeably better than rate control. Pressure-based control, however, clearly provided better performances in terms of Euclidean distances-even though this difference could not be found for the angular distances. We can thus partially confirm our hypothesis H4: the overall performance obtained by pressure-based control is better than the three other conditions, but the difference (compared to slider-based and speed-based control) is small and only noticeable for Euclidean distances.

\section{Fatigue}

We can confirm our hypothesis H5: pressure-based control caused more fatigue in participants' fingers than the other three interaction techniques. However, for hand, arm, and shoulder fatigue the pressure-based control caused only as much fatigue or less compared to the other techniques we tested. Hypothesis H5 should thus be treated in a nuanced way. This point is further reinforced by the overall and weighted overall fatigue ratings we showed in Fig. 10 that exhibit no evidence for pressure-based control causing more fatigue than the other techniques overall. We can thus conclude that fatigue considerations do not prevent us using pressure-based control. Moreover, we conjecture that finger fatigue could have been reduced with if the pressure sensors were better integrated in the device: In our study the participants pressed their fingers on a bare and hard pressure sensor attached to the tablet. The use of memory foam or other visco-elastic components above the pressure sensors could probably reduce the finger fatigue.

We can, however, dismiss our hypothesis H6. Not only was the mental workload of the speed-based control similar to that of the other techniques (or smaller than that of rate control), but also the overall fatigue it caused was not higher than the fatigue of the other techniques-except potentially compared to pressure-based control in the weighted average.

\section{Workload}

While the individual workload aspects may each be slightly different, the overall workload is lower for pressure-based control than for all the other techniques. This result only further supports our conclusion about the performance measurements and fatigue ratings, showing that pressure-based control is not only a viable contender but an ideal choice for the adjustment of the gain factor. Moreover, the perceived performance (Fig. 7(d)) was best with pressure-based control. This specific result means that our participants were not only more precise with this technique but also had the highest confidence in their results in this experimental condition.

\section{Preferences}

In addition to all these measured benefits, our experimental results also allow us to conclude that pressured-based control was clearly the favorite technique to adjust the gain factor among our 24 participants. Indeed, 15 of them reported that it was their preferred technique. Such a ratio allows us to generalize our results beyond the pool of our 24 participants: overall, people will likely prefer the pressure-based control over the other tested techniques.

\section{Design Guidelines and Recommendations}

While pressure-based control thus has clear benefits to controlling the gain factor, it is sometimes also necessary to set a well-defined gain factor value. Such control is difficult with pressure input, but is easily facilitated by a slider or text widget as highlighted by three participants. So while we advocate the use of pressure input in general, it may be useful to integrate it with a way to set an explicit value though a slider or otherwise, for example for expert users or in professional tools. In fact, 
nothing prohibits the implementation of all four techniques, with a possibility to set the main technique based on personal preferences. Yet, we still recommend to use pressure-based control as the default due to its discussed performance benefits.

\section{Limitations}

Despite our careful experimental design there are some limitations to be mentioned. In particular, there may be a bias towards the novelty of the pressure-based control-after all, it has only recently been introduced to mobile device interaction for the general public. We also argue, however, that the overall tangible manipulation was new as well to our participants and that, therefore, the novelty effect of pressure-based manipulation was likely not too large. While no participant reported being tired or feeling any particular fatigue in our first study which exposed them to pressure manipulation for 20 minutes, we-for the sake of performance evaluation-reduced the interaction time for each technique to approximately 6 minutes. This reduction implies that further work is needed to check the influence of each technique on fatigue or physical demand with a longer exposition time. In addition, the task we asked our participant to carry out was a generic 3D manipulation task. While it is used in many 3D studies, it may not completely resemble the $3 \mathrm{D}$ interaction tasks needed for specific 3D interaction scenarios. Pressure-based control should thus also be evaluated in the context other application domains, including those that rely on less abstract tasks than 3D docking.

\section{CONCLUSION}

With our design and evaluation of a pressure-based interactive control of the gain factor for 3D navigation we identified an appropriate channel for such manipulations-one that is independent from the otherwise dominant channels such as tactile and tangible input. Our first experiment guided our interaction design and the specific pressure mapping we used, while the second experiment provided clear evidence for the advantages of the new design over other types of input.

One of the most important insights we derive from our experiments is that the use of pressure input allowed our participants, in particular, to focus on their 3D manipulation task without the need to constantly reflect the interaction mapping (as it was the case for speed-based and rate control) and without the need to constantly change their interaction focus to be able to interact with a separate widget on the display (like it was necessary for slider-based control). We argue that our participants were thus more effective in their interaction (better performance), without pressure-based control causing any additional cost on workload or fatigue.

In our work we used a specific back-of-device design to enable pressure-based interaction. While we believe that this setup has advantages with respect to the ergonomics of the interaction with the tablet and the use of screen real estate, the general use of pressure as an input channel for gain factor control does not require the use of such a back-of-device design. Input could thus also be provided on the front of the device-and there are already commercially available devices that offer such display-based pressure sensing. Moreover, due to the specific character of the gain factor control that only requires the input of differences - and not of precise, absolute input values-it is also possible to use existing pseudo-pressure sensing $[1,2]$ which could be explored in the future.

Our work thus provides guidelines to implement gain factor control for 3D manipulations and other forms of interaction, for a variety of mobile devices. Our results, however, may also be generalizable to the control of other scalar values where only relative changes are important and constant visual feedback is provided.

\section{REFERENCES}

1. Ahmed Sabbir Arif, Ali Mazalek, and Wolfgang Stuerzlinger. 2014. The use of pseudo pressure in authenticating smartphone users. In Proceedongs of the International Conference on Mobile and Ubiquitous Systems: Computing, Networking and Services. ICST, Brussels, 151-160. DOI :

http: //dx . doi . org/10.4108/icst . mobiqui tous . 2014 . 257919

2. Ahmed Sabbir Arif and Wolfgang Stuerzlinger. 2013. Pseudo-pressure detection and its use in predictive text entry on touchscreens. In Proceedings of the Australian Computer-Human Interaction Conference: Augmentation, Application, Innovation, Collaboration. ACM, New York, 383-392. DOI :

http://dx. doi . org/10.1145/2541016.2541024

3. Thom Baguley. 2009. Standardized or simple effect size: What should be reported? British Journal of Psychology 100, 3 (Aug. 2009), 603-617. D0I : http://dx.doi .org/10.1348/000712608X377117

4. Monya Baker. 2015. Statisticians issue warning over misuse of P values. Nature 531, 7593 (March 2015), 151. DOI : http://dx.doi .org/10.1038/nature.2016.19503

5. Patrick Baudisch and Gerry Chu. 2009. Back-of-device interaction allows creating very small touch devices. In Proceedings of the SIGCHI Conference on Human Factors in Computing Systems. ACM, New York, 1923-1932. DOI :

http://dx.doi .org/10.1145/1518701.1518995

6. Hrvoje Benko, Andrew D. Wilson, and Patrick Baudisch. 2006. Precise selection techniques for multi-touch screens. In Proceedings of the SIGCHI Conference on Human Factors in Computing Systems. ACM, New York, 1263-1272. DOI :

http://dx. doi . org/10.1145/1124772.1124963

7. Lonni Besançon, Paul Issartel, Mehdi Ammi, and Tobias Isenberg. 2017. Hybrid tactile/tangible interaction for 3D data exploration. IEEE Transactions on Visualization and Computer Graphics 23, 1 (Jan. 2017), 881-890. D0I : http://dx. doi . org/10.1109/TVCG. 2016.2599217

8. Renaud Blanch, Yves Guiard, and Michel Beaudouin-Lafon. 2004. Semantic pointing: Improving target acquisition with control-display ratio adaptation. In Proceedings of the SIGCHI Conference on Human Factors in Computing Systems. ACM, New York, 519-526. DOI : http://dx. doi . org/10.1145/985692 . 985758 
9. Stephen A. Brewster and Michael Hughes. 2009. Pressure-based text entry for mobile devices. In Proceedings of the SIGCHI Conference on Human Factors in Computing Systems. ACM, New York. DOI : http://dx.doi.org/10.1145/1613858.1613870

10. Neil Burgess, Hugo J. Spiers, and Eleni Paleologou. 2004. Orientational manoeuvres in the dark: Dissociating allocentric and egocentric influences on spatial memory. Cognition 94, 2 (Dec. 2004), 149-166. DOI : http://dx. doi .org/10. 1016/j . cognition. 2004.01.001

11. Bill Buxton. 2007. Multi-touch systems that I have known and loved. Website. (Jan. 2007).

http://www. billbuxton. com/multitouch0verview.html Updated last on June 12, 2014, visited in March 2016.

12. William Buxton, Ralph Hill, and Peter Rowley. 1985. Issues and techniques in touch-sensitive tablet input. ACM SIGGRAPH Computer Graphics 19, 3 (July 1985), 215-224. DOI: http://dx. doi.org/10.1145/325165.325239

13. Géry Casiez, Daniel Vogel, Ravin Balakrishnan, and Andy Cockburn. 2008. The impact of control-display gain on user performance in pointing tasks. Human-Computer Interaction 23, 3 (2008), 215-250. DOI : http://dx. doi.org/10.1080/07370020802278163

14. Jared Cechanowicz, Pourang Irani, and Sriram Subramanian. 2007. Augmenting the mouse with pressure sensitive input. In Proceedings of the SIGCHI Conference on Human Factors in Computing Systems. ACM, New York, 1385-1394. DOI : http: //dx . doi .org/10.1145/1240624.1240835

15. Robert Coe. 2002. It's the effect size, stupid: What effect size is and why it is important. In Proceedings of the Annual Conference of the British Educational Research Association.

http : //www . leeds . ac . uk/educol/documents/00002182 . htm

16. Geoff Cumming. 2014. The new statistics: Why and how. Psychological Science 25, 1 (Jan. 2014), 7-29. DOI: http://dx. doi . org/10.1177/0956797613504966

17. Nicola Dell, Vidya Vaidyanathan, Indrani Medhi, Edward Cutrell, and William Thies. 2012. "Yours is better!" Participant response bias in HCI. In Proceedings of the SIGCHI Conference on Human Factors in Computing Systems. ACM, New York, 1321-1330. DOI : http://dx.doi.org/10.1145/2207676.2208589

18. Pierre Dragicevic. 2016. Fair statistical communication in HCI. In Modern Statistical Methods for HCI, Judy Robertson and Maurits Kaptein (Eds.). Springer International Publishing, Cham, Switzerland, Chapter 13, 291-330. DOI :

http : //dx . doi .org/10 . 1007/978-3-319-26633-6_13

19. Pierre Dragicevic, Fanny Chevalier, and Stéphane Huot. 2014. Running an HCI experiment in multiple parallel universes. In Extended Abstracts on Human Factors in Computing Systems. ACM, New York, 607-618. DOI : http://dx. doi . org/10.1145/2559206.2578881
20. James D. Foley, Victor L. Wallace, and Peggy Chan. 1984. The human factors of computer graphics interaction techniques. IEEE Computer Graphics and Applications 4, 11 (Nov. 1984), 13-48. DOI:

http://dx.doi.org/10.1109/MCG. 1984.6429355

21. Clifton Forlines, Chia Shen, and Bill Buxton. 2005. Glimpse: A novel input model for multi-level devices. In Extended Abstracts on Human Factors in Computing Systems. ACM, New York, 1375-1378. DOI :

http://dx.doi.org/10.1145/1056808.1056920

22. Scott Frees and G. Drew Kessler. 2005. Precise and rapid interaction through scaled manipulation in immersive virtual environments. In Proceedings of the IEEE Conference on Virtual Reality. IEEE, 99-106. DOI : http://dx. doi . org/10.1109/VR. 2005.1492759

23. Sandra G. Hart. 2006. NASA-task load index (NASA-TLX); 20 years later. Proceedings of the Human Factors and Ergonomics Society Annual Meeting 50, 9 (Oct. 2006), 904-908. DOI :

http://dx.doi.org/10.1177/154193120605000909

24. Christopher F. Herot and Guy Weinzapfel. 1978. One-point touch input of vector information for computer displays. ACM SIGGRAPH Computer Graphics 12, 3 (Aug. 1978), 210-216. DOI : http://dx.doi.org/10.1145/965139.807392

25. Ken Hinckley, Randy Pausch, John C. Goble, and Neal F. Kassell. 1994. A survey of design issues in spatial input. In Proceedings of the Annual ACM Symposium on User Interface Software and Technology. ACM, New York, 213-222. DOI : http://dx. doi .org/10.1145/192426.192501

26. Shigeo Hiraoka, Isshin Miyamoto, and Kiyoshi Tomimatsu. 2003. Behind touch, a text input method for mobile phones by the back and tactile sense interface. In Proceedings of Interaction. Information Processing Society of Japan, Tokyo, 131-138.

27. Hiroshi Ishii and Brygg Ullmer. 1997. Tangible bits: Towards seamless interfaces between people, bits and atoms. In Proceedings of the SIGCHI Conference on Human Factors in Computing Systems. ACM, New York, 234-241. DOI : http://dx . doi .org/10.1145/258549.258715

28. Paul Issartel, Lonni Besançon, Florimond Guéniat, Tobias Isenberg, and Mehdi Ammi. 2016a. Preference between allocentric and egocentric 3D manipulation in a locally coupled configuration. In Proceedings of the ACM Symposium on Spatial User Interaction. ACM, New York, 79-88. DOI : http://dx. doi .org/10.1145/2983310.2985750

29. Paul Issartel, Lonni Besançon, Tobias Isenberg, and Meryem Ammi. 2016b. A tangible volume for portable 3D interaction. In Proceedings of the International Symposium on Mixed and Augmented Reality. IEEE Computer Society, Los Alamitos.

30. Paul Issartel, Florimond Guéniat, Tobias Isenberg, and Mehdi Ammi. 2016c. Analysis of locally coupled 3D manipulation mappings based on mobile device motion. 
arXiv.org preprint 1603.07462.

http://www . arxiv. org/abs/1603.07462

31. Jeroen Keijser, Sheelagh Carpendale, Mark Hancock, and Tobias Isenberg. 2007. Exploring 3D interaction in alternate control-display space mappings. In Proceedings of the IEEE Symposium on 3D User Interfaces. IEEE Computer Society, Los Alamitos, 17-24. DOI :

http: //dx . doi . org/10 . 1109/3DUI . 2007 . 340769

32. Roberta L. Klatzky. 1998. Allocentric and egocentric spatial representations: Definitions, distinctions, and interconnections. In Spatial cognition, Christian Freksa, Christopher Habel, and Karl F. Wender (Eds.). Springer, Berlin/Heidelberg, 210-216. DOI :

http://dx. doi .org/10.1007/3-540-69342-4_1

33. Martin Krzywinski and Naomi Altman. 2013. Points of significance: Error bars. Nature Methods 10, 10 (Oct. 2013), 921-922. http: //dx. doi.org/10. 1038/nmeth. 2659

34. Kevin A. Li, Patrick Baudisch, and Ken Hinckley. 2008. Blindsight: Eyes-free access to mobile phones. In Proceedings of the SIGCHI Conference on Human Factors in Computing Systems. ACM, New York, 1389-1398. DOI :

http://dx.doi.org/10.1145/1357054.1357273

35. Hai-Ning Liang, Cary Williams, Myron Semegen, Wolfgang Stuerzlinger, and Pourang Irani. 2013. An investigation of suitable interactions for $3 \mathrm{D}$ manipulation of distant objects through a mobile device. International Journal of Innovative Computing, Information and Control 9, 12 (Dec. 2013), 4737-4752.

http://www.ijicic.org/apchi12-291.pdf

36. I. Scott MacKenzie and Stan Riddersma. 1994. Effects of output display and control-display gain on human performance in interactive systems. Behaviour \& Information Technology 13, 5 (1994), 328-337. DOI : http://dx . doi . org/10.1080/01449299408914613

37. Ross McLachlan, Daniel Boland, and Stephen Brewster. 2014. Transient and transitional states: pressure as an auxiliary input modality for bimanual interaction. In Proceedings of the SIGCHI Conference on Human Factors in Computing Systems. ACM, New York, 401-410. DOI :

http://dx.doi . org/10.1145/2556288.2557260

38. Ross McLachlan and Stephen Brewster. 2015. Bimanual input for tablet devices with pressure and multi-touch gestures. In Proceedings of the International Conference on Human-Computer Interaction with Mobile Devices and Services. ACM, New York, 547-556. DOI: http://dx.doi .org/10.1145/2785830.2785878

39. Jakob Nielsen. 1993. Usability Engineering. Morgan Kaufmann, San Francisco. DOI : http://dx . doi . org/10 . 1016/B978-0-08-052029-2 . 50001-2

40. Ivan Poupyrev, Suzanne Weghorst, Mark Billinghurst, and Tadao Ichikawa. 1998. Egocentric object manipulation in virtual environments: Empirical evaluation of interaction techniques. Computer Graphics Forum 17, 3 (Sept. 1998), 41-52. DOI :

http://dx. doi . org/10.1111/1467-8659.00252

41. Mahfuz Rahman, Sean Gustafson, Pourang Irani, and Sriram Subramanian. 2009. Tilt techniques: investigating the dexterity of wrist-based input. In Proceedings of the SIGCHI Conference on Human Factors in Computing Systems. ACM, 1943-1952.

42. Gonzalo Ramos and Ravin Balakrishnan. 2005. Zliding: Fluid zooming and sliding for high precision parameter manipulation. In Proceedings of the Annual ACM Symposium on User Interface Software and Technology. ACM, New York, 143-152. D0I :

http://dx. doi .org/10.1145/1095034.1095059

43. Gonzalo Ramos, Matthew Boulos, and Ravin Balakrishnan. 2004. Pressure widgets. In Proceedings of the SIGCHI Conference on Human Factors in Computing Systems. ACM, New York, 487-494. DOI :

http://dx.doi .org/10.1145/985692.985754

44. Xiangshi Ren, Jibin Yin, Shengdong Zhao, and Yang Li. 2007. The adaptive hybrid cursor: A pressure-based target selection technique for pen-based user interfaces. In Proceedings of the IFIP Conference on

Human-Computer Interaction. Springer,

Berlin/Heidelberg, 310-323. DOI :

http: //dx . doi . org/10 . 1007/978-3-540-74796-3_29

45. Carsten Schwesig, Ivan Poupyrev, and Eijiro Mori. 2004. Gummi: A bendable computer. In Proceedings of the SIGCHI Conference on Human Factors in Computing Systems. ACM, New York, 263-270. D0I : http://dx. doi . org/10.1145/985692.985726

46. Christopher D. Shaw. 1998. Pain and fatigue in desktop VR: Initial results. In Proceedings of Graphics Interface. CIPS, Toronto, 185-192. DOI : http: //dx. doi . org/10.20380/GI1998.23

47. Erh-li Early Shen, Sung-sheng Daniel Tsai, Hao-hua Chu, Yung-jen Jane Hsu, and Chi-wen Euro Chen. 2009. Double-side multi-touch input for mobile devices. In Extended Abstracts on Human Factors in Computing Systems. ACM, New York, 4339-4344. DOI : http://dx.doi.org/10.1145/1520340.1520663

48. Craig Stewart, Michael Rohs, Sven Kratz, and Georg Essl. 2010. Characteristics of pressure-based input for mobile devices. In Proceedings of the SIGCHI Conference on Human Factors in Computing Systems. ACM, New York, 801-810. DOI :

http://dx. doi .org/10.1145/1753326.1753444

49. Masanori Sugimoto and Keiichi Hiroki. 2006. HybridTouch: An intuitive manipulation technique for PDAs using their front and rear surfaces. In Proceedings of the International Conference on Human-Computer Interaction with Mobile Devices and Services. ACM, New York, 137-140. DOI :

http://dx. doi .org/10.1145/1152215.1152243 
50. Philip Tuddenham, David Kirk, and Shahram Izadi. 2010. Graspables revisited: Multi-touch vs. tangible input for tabletop displays in acquisition and manipulation tasks. In Proceedings of the SIGCHI Conference on Human Factors in Computing Systems. ACM, New York, 2223-2232. DOI:

http://dx. doi.org/10.1145/1753326.1753662

51. Gary R. VandenBos (Ed.). 2009. Publication Manual of the American Psychological Association (6 ${ }^{\text {th }}$ ed.). American Psychological Association, Washington, DC. http://www. apastyle. org/manual/

52. Daniel Wigdor, Clifton Forlines, Patrick Baudisch, John Barnwell, and Chia Shen. 2007. LucidTouch: A see-through mobile device. In Proceedings of the Annual ACM Symposium on User Interface Software and Technology. ACM, New York, 269-278. DOI: http://dx. doi . org/10.1145/1294211.1294259

53. Daniel Wigdor, Darren Leigh, Clifton Forlines, Samuel Shipman, John Barnwell, Ravin Balakrishnan, and Chia Shen. 2006. Under the table interaction. In Proceedings of the Annual ACM Symposium on User Interface Software and Technology. ACM, New York, 259-268. DOI:http://dx.doi.org/10.1145/1166253.1166294

54. Graham Wilson, Craig Stewart, and Stephen A. Brewster. 2010. Pressure-based menu selection for mobile devices. In Proceedings of the International Conference on Human-Computer Interaction with Mobile Devices and Services. ACM, New York, 181-190. DOI:

http: //dx . doi .org/10.1145/1851600. 1851631

55. Graham Alasdair Wilson. 2013. Using pressure input and thermal feedback to broaden haptic interaction with mobile devices. Ph.D. Dissertation. University of Glasgow, Scotland.

http: //theses.gla.ac.uk/id/eprint/4363

56. Aileen Worden, Nef Walker, Krishna Bharat, and Scott Hudson. 1997. Making computers easier for older adults to use: Area cursors and sticky icons. In Proceedings of the SIGCHI Conference on Human Factors in Computing Systems. ACM, New York, 266-271. DOI:

http://dx . doi .org/10 . 1145/258549 . 258724

57. Shumin Zhai. 1998. User performance in relation to 3D input device design. ACM SIGGRAPH Computer Graphics 32, 4 (Nov. 1998), 50-54. DOI:

http: //dx . doi .org/10 . 1145/307710. 307728 